\title{
Negotiating styles adopted by consultant psychiatrists when prescribing antipsychotics
}

\author{
Robert Chaplin, Paul Lelliott, Alan Quirk \& Clive Seale
}

\begin{abstract}
A good therapeutic alliance between mental health professionals and patients with psychosis can enhance adherence to medication regimens and improve clinical outcome. This article explores how the therapeutic alliance might be developed with respect to decisions to prescribe antipsychotic medication. It does this by presenting the implications for practice that arise from a recent qualitative interview study with consultant psychiatrists. We consider strategies for strengthening the therapeutic alliance, occasions when it might be appropriate to suspend shared decision-making temporarily, techniques used to enable discussion of symptoms and side-effects, and how issues of adherence are uncovered and addressed. Psychiatrists already possess considerable skills in these areas. The dissemination of these to colleagues forms an important opportunity for CPD.
\end{abstract}

Psychiatrists have been urged to practise 'patientcentred psychiatry' (Bhugra \& Holsgrove, 2005). This approach requires an enhanced doctor-patient relationship, which includes the creation of common ground between the two. Few studies have examined psychiatrists' consultation styles or attempted to identify styles and approaches that improve the therapeutic alliance between psychiatrists and their patients. This article presents additional analysis of the results of our recent study in which consultant psychiatrists were interviewed about practices adopted when prescribing antipsychotic medication (Seale et al, 2006). We present the findings in the hope that this will enable psychiatrists to reflect on and enhance their consultation styles by learning from the methods employed by other consultant colleagues. More specifically, we will focus on the strategies that consultant psychiatrists currently say they use to cultivate the therapeutic alliance, explain side-effects, uncover and address non-adherence and deal with the need to make decisions about compulsory treatment.

Many of the challenges that psychiatrists face in maintaining a therapeutic alliance with patients with psychosis are no different from those involved in working with patients with other chronic mental health problems. These include the need for a long-term therapeutic relationship, changes in capacity and insight, and the possibility of making compulsory decisions under the Mental Health Act 1983. The findings from this study might therefore be relevant to the care of patients prescribed other classes of drugs or with other disorders. There are two important things to note when reading this article. First, our study was not a survey of actual consultations: psychiatrists may deviate in real life from the practices they espouse. Second, it provides only suggestions about working practices; there are no clear right or wrong ways of working and some of the practices described here may seem inappropriate to some readers.

\section{Improving the therapeutic alliance}

The conduct of encounters with patients is built up from clinical experience arising from years of training, observation of senior colleagues and experimentation. It is perhaps less likely than other aspects of practice to be influenced by research or

Robert Chaplin is a research fellow at the Royal College of Psychiatrists' Research and Training Unit (CRTU) (21 Mansell Street, London E1 8AA, UK. Email: rchaplin@cru.rcpsych.ac.uk) and a consultant in general adult psychiatry at Oxfordshire Mental Healthcare NHS Trust. He has interests in audit and learning disability. Paul Lelliott is Director of the CRTU and a consultant psychiatrist employed by Oxleas NHS Trust, where he works as a member of a community mental health team. Alan Quirk is a research fellow at the CRTU. As a research sociologist he has used qualitative methods to study psychiatrist-patient communication in a range of situations, including Mental Health Act assessments, ward rounds on acute wards and out-patient consultations. Clive Seale is a professor of sociology at Brunel University. He is author of many books and articles on aspects of medical sociology and social research methods. He is presently pursuing projects in the fields of psychiatry, end-of-life care and treatment of health issues by the mass media. 
to be evidence based. We use the term therapeutic alliance to denote the central issue that this article addresses. Other terms that convey the same concept include helping alliance, therapeutic relationship and working alliance. A therapeutic alliance is achieved when the participants have an open, trusting and collaborative relationship (Frank \& Gunderson, 1990) that has healing properties (McCabe \& Priebe, 2004).

Most of the research aimed at improving consultation style has been in general practice. For example, educational interventions to improve consultation style have led to better practice by reducing the rate of prescribing of unnecessary medications for people with sore throats. A similar attempt to improve outcome of patients with depression in primary care by changing the style of consultations has produced more mixed results (Gask et al, 2004). We are not aware of any study that has investigated the impact of training on psychiatrists' relationships with patients or on their prescribing behaviour. It is hoped that this article might contribute to the debate about what such training might consist of and what it might achieve.

\section{Therapeutic alliance and outcome in patients with psychosis}

Lacro et al's (2002) review of studies identified possible reasons for poor adherence to antipsychotic medication regimens and for variation in its reported prevalence. These include factors related to the patient such as poor insight, negative attitudes to medication, previous non-adherence and shorter duration of illness. The quality of caregiving can also influence adherence; for example, poor discharge planning has a negative effect. A good therapeutic alliance between psychiatrists and patients with schizophrenia has been shown to increase adherence to antipsychotic medication regimens (Frank \& Gunderson, 1990). Furthermore, Priebe \& Gruyters (1993) showed that, for a group of psychiatric patients most of whom had schizophrenia, a favourable perception of the alliance predicted a good outcome at 20 months. The same authors investigated the effects of a session with their doctor and keyworker that allowed a small subgroup of day hospital patients with schizophrenia who were dissatisfied with their treatment to express their views about it (Priebe \& Gruyters 1999). Those randomised to receive the extra session had modest wishes which, when met, improved treatment satisfaction and slightly improved psychopathology compared with a control group who received treatment as usual.

\section{Talking about psychotic symptoms}

Many people with schizophrenia want to discuss their symptoms when they consult a psychiatrist. McCabe et al (2002) analysed a series of tape-recorded out-patient consultations involving patients with schizophrenia and their psychiatrists, and found that psychiatrists are sometimes reluctant to engage with patients who initiate talk about their experiences of delusions and auditory hallucinations. This is a potential area for a failure of communication or, more specifically, a failure to elicit patients' concerns. When psychiatrists wish to initiate a conversation about psychotic symptoms, they often feel it prudent to enquire very carefully, using the same language as the patient. This is illustrated by the examples below, which are taken from our interviews with psychiatrists (certain extracts reprinted with permission of Elsevier from Seale et al, 2006).

'You do have to ask the closed questions too, but there's ways of doing it. It's about sort of fitting them in, in amongst the things that maybe the patient wants to talk about, which isn't always their voices ... young psychotic men often have their own way of describing their own experiences and one way to upset them is for a new psychiatrist to come in and say "Well, how are the voices at the moment?" And it can often be a reason for not coming back'.

An attempt to empathise with the patient's psychotic experience is shown in the following:

'I think I try to get some idea of the person's experience and feelings about illness and the way they think about it ... I will sometimes use words that the person themselves has used, or refer to some experience that they told me about, perhaps some time ago, and try to build that in, so I hope the whole thing makes more sense to the person'.

\section{Diagnosis}

The concept of diagnosis has a central role in decisions about the treatment of patients; for example, most clinical practice guidelines published by the National Institute for Health and Clinical Excellence (NICE) are about a diagnosis or cluster of diagnoses. It also features prominently in the training of psychiatrists. In England, psychiatrists are required to code diagnosis and it is likely that the diagnosis will be revealed to patients as a result of the requirement that letters to general practitioners are copied to patients (Department of Health, 2003). However, the psychiatrists who were interviewed did not consider that discussion of diagnosis was of particular importance in consultations with patients 
prescribed antipsychotic medication. In fact, some viewed the giving of a diagnosis as anti-therapeutic and itself a risk of generating conflict:

'If someone has, say, delusions and they feel the devil is chasing after them or something, if you say to someone "You have schizophrenia" you're going to clash against their own belief'.

One possible approach, as illustrated by the following extract, is to introduce the concept of diagnosis by asking the patient's views first and to follow that with the patient's own explanation:

'I'm always happy to give them the diagnostic words but I would usually say "You know what's the matter with you, you can describe what's the matter with you better than I can. This is the word that us doctors use for what you've got"'.

The psychiatrist might circumvent the need to refer to a diagnosis by instead talking about specific symptoms or problems in an area of functioning that might be helped by medication:

'The challenge is to find common ground, do they believe they have an area of difficulty that ... might affect their concentration, or their ability to do what their brother's doing or hold down a job? Then you have to build up from the common difficulty that you both agree on to the point where medication's accepted'.

An alternative approach is to explain the symptoms in order to normalise or minimise the impact of receiving a diagnosis of mental illness:

'Taking medication is a sign that in fact your mind might not be working well. That's a very difficult thing to take on board. So what I say to patients now is something around the lines of "A part of your mind is not working"'

One psychiatrist did not consider it essential to be in agreement with the patient about the exact diagnosis. It is even possible for there to be an explicit disagreement without this resulting in conflict:

'We may ... agree to differ on the nature of the illness or the psychosis but agree a trial of medication that might help with sleep, or something like that'.

Informing a patient about their diagnosis needs to be timed carefully and handled sensitively:

'Some patients, I would probably say straight away "Look, you've got a disorder which is called schizoaffective disorder, schizophrenia". Other patients, I probably won't say it, unless they ask. But if they ask, I would always tell the diagnosis... Yes, I would give the diagnosis, but not always the first time'.

In summary, psychiatrists need to exercise considerable skill in deciding the extent to which diagnosis should be discussed, the exact language to be used and the timing of the discussion. The aim is to match their own conceptual models of illness with the needs of individual patients, who have varying degrees of insight and different needs at different phases of their illness.

\section{Shared decision-making and the therapeutic alliance}

Psychiatrists rate the formation of a therapeutic alliance as fundamentally important when treating people with antipsychotic medication:

'I genuinely see us as in it together, if you like, that it's a partnership and it's not going to work if I am somehow in a position of superiority and they're in a subordinate subject position. I think it just won't work'.

Some ways of achieving this alliance are outlined in Box 1. The quotations below, again from our interview study with psychiatrists, illustrate techniques in achieving shared decision-making with patients. The first is an example of how a psychiatrist described attempting to strike a bargain with a patient who was reluctant to continue antipsychotic medication:

'He decided that he wanted to come off medication, and I discussed it with him and he was continuing to use cannabis, and he was eventually insistent that he was going to stop it [the medication] and I said I would allow it. As a quid pro quo perhaps he would agree to stop using cannabis'.

Box 1 Techniques for establishing a therapeutic alliance

- Listening to the individual's views about their situation, their illness and their medications

- Showing empathy, understanding, warmth, encouragement, respect and closeness

- Using language carefully and tactfully, using non-technical words and explanations the individual can understand or that reflect their way of seeing things

- Knowing the individual over a long period

- Expressing a 'human' rather than a purely professional response

- Overcoming hostility, suspicion or conflict

- Tolerating disagreement

- Not looking rushed

- Giving the individual time to process and respond to information and reach decisions

- Striking bargains

- Displaying knowledge of the individual's personal background, circumstances and preferences

- Using humour 
In the following extract a psychiatrist shows the need to tolerate and feel comfortable about disagreement when it is not possible to reach agreement with a patient:

'I just think he's the one having the injections in the end, he hates them so much, we'll just have to go for something else'.

Remaining calm, giving patients time and not appearing rushed were all seen as important. These can be understood as the avoidance of a relationship involving high expressed emotion (Tattan \& Tarrier, 2000). Finally, the quality of 'being human' with patients is shown below by a psychiatrist who wanted to give the impression of providing a personal service:

'I do realise that it's a deception, but it's a way of making the patient think that I have a personal recollection of their personal situation - I'll jot down the names of their children or whatever [in the notes] so that I'm able to at least have a person think that I know something about their personal life'.

The judicious use of humour is seen by another psychiatrist as further enhancing the therapeutic alliance where a good alliance already exists:

'I will write what I am wanting the person to have on a compliments slip so that they can show it to the GP or the receptionist ... sometimes I might put a little joke on it, so its more of a personal message'.

\section{Departures from shared decision-making}

Psychiatrists may have temporarily to suspend shared decision-making in emergencies and when patients lack capacity to make treatment decisions. However, they report a variety of strategies for doing this and managing the consequences. In the case below, and somewhat controversially, the psychiatrist reports being untruthful with a patient in order attempt to create a therapeutic alliance:

'[A man] who had schizophrenia but was convinced he did not have a mental health problem of any type and really didn't want to be seen ... he really didn't see the point in seeing anybody, so I called at the house without warning in advance, or else he might have left, ... apologised and said "It's all part of system followup", trying to locate the blame in some bureaucratic system, and said "I really apologise. Now I'm here, can we talk these things through?"'.

Other controversial issues relating to the therapeutic alliance are presented in Box 2. Within the therapeutic alliance psychiatrists accept that it is at times necessary to take more control in the making of treatment decisions or to become directive.

\section{Box 2 Controversial issues}

- Research has shown that psychiatrists can find it hard to respond to patients' psychotic symptoms

- Whether it is ever ethically justified to be untruthful with a patient in order to generate a therapeutic alliance

- When to be directive or even coercive

- Discussing a patient's diagnosis can be seen to present problems for the therapeutic alliance

- Psychiatrists have differing opinions about the appropriate time to discuss side-effects with patients

- Psychiatrists may be able to improve their interactions with patients by reflecting on their individual styles

'Sometimes one has to act in a slightly paternalistic way, that you think, well I know this patient needs this. I know it's going to make a major difference to their lives and the chance of them getting these things is probably quite small so lets try and go with it - and again it depends on the patient'.

The use of a directive style by psychiatrists might be seen by others as potentially coercive, as shown by the extract below:

'I sometimes have to say really tough things to people ... where I'm going to have to say to them "Look, you're running away from this issue and you're going to go on being stuck unless you deal with this a different way"'.

The following extract outlines a situation where a psychiatrist anticipates employing a coercive approach:

'You would like the individual to be aware that there are options but you also want them to fully understand the implications of not agreeing. So that whereas in the first part of the interview you might be presenting the case more from the positive gains, it might be that later on in the interview if you feel that they are not keen to go down that route you then might have to bring in the realities ... I wouldn't immediately move into a sort of coercive "You will do this because I am the doctor" strategy, but it might be that you then say "Well, it's obviously your decision but it might be that if we don't get on top of it quickly that you're going to end up in hospital or have to stop work for a while", so you want them to be fully aware of the implication of not going on with the medication plan'.

The importance of being explicit about the possible use of coercion was stressed by another psychiatrist: 
'If it gets to the stage when you're going to have to force them I'd rather be straight about that as well... I think she knows I'm straight ... and she's agreed that if she does relapse she'll come and see me and we'll admit her voluntarily'.

Nevertheless, coercion was seen as reflecting a temporary failure of collaborative methods where a return to shared decision-making would be attempted once the patient's clinical state had improved:

'He's on a depot injection now and he's got a lot of objections to it ... and in fact I'm not going to carry on with it ... he has decided against this medication... He's now well enough to make a reasonable argument about it all ... and I think in the longer run it's really the only way'.

Finally, this psychiatrist illustrated the importance, when confronted with a situation in which coercion is being used, to remain patient and avoid conflict:

'I felt I had to be very firm, very direct with him: "I need to know are you going to think about this, have you thought about it? Are you going to think about it?" and the best decision I got from him after about ten minutes of standing, I actually tried to stop myself laughing, realising how ridiculous this was, he eventually said that he would tell me next week'.

\section{Side-effects}

When enquiring about side-effects, the following psychiatrist stressed the importance of not assuming that the patient will attribute the presence of new physical symptoms to the medication:

'I think one of the problems is that we need to find out about the side-effects. Quite often patients don't identify certain symptoms as side-effects so they need to be enquired about in a more systematic way rather than just asking someone if they've got side-effects'.

Although psychiatrists reported that side-effects were more likely than anything else to lead to nonadherence, they encountered many problems in discussing them without adversely affecting their patients' motivation to take medications:

'If you emphasise too much the side-effects sometimes you feel that you are increasing the probability of the person saying “No, I don't want to take this medication"' '.

However, many studies of this aspect of patient education have failed to show that it has any adverse effects on outcome (e.g. Chaplin \& Kent, 1998). Other psychiatrists preferred to be frank at the beginning of treatment:

'The best way of dealing with it is actually pre-empting the things, tell them from the outset. I try to, but you know, the most common side-effects, I'm sure you are aware, are weight gain, effect on libido, feeling tired and so on, I try to pre-empt that'.

Another complicating factor is the patient's capacity to be able to make an informed decision about treatment. To this psychiatrist, a potential solution was to defer the discussion about sideeffects to a later date:

'I try to engage the person and get them on medication to the point that they are no longer psychotic and then I have a more in-depth discussion'.

Other barriers to discussion of side-effects include perceived limits of psychiatrists' knowledge about all possible side-effects and the impracticality of discussing every single one:

'You can't say all of the potential side-effects, because some of them are very rare'.

Smith \& Henderson (2000), using a postal questionnaire, revealed that psychiatrists were selective about which side-effects they discussed. Laugharne et al (2004) found considerable international variation between psychiatrists in the extent to which they discussed tardive dyskinesia with patients. It seems likely that there is individual variation in the discussion of side-effects, although no recent studies have reported on this. A potential solution to the need for disclosure was suggested in the routine provision of written information about medication and advice on the internet:

'I don't literally intentionally bombard them but you can give them a lot of information about the sideeffects... That can be quite overwhelming so you have to have patience to simply allow them to go away, or ask them to go away and think about it. You send them away with written information and websites'.

In conclusion, practice in informing patients about side-effects is influenced by many factors, including the types of side-effects, individual variations in practice, the severity of the patient's illness and the patient's needs. In a recent study, however, $50 \%$ of patients claimed to have received no information at all before starting antipsychotic medication (Olofinjana \& Taylor, 2005). There is a need for observational studies to investigate the extent to which side-effects are discussed and practical methods for discussing them.

\section{Non-adherence}

Non-adherence was seen by psychiatrists as a central problem that frequently involved taking too much or inappropriate medication as well as not taking it. A calm and non-confrontational approach was advocated by this psychiatrist: 


\section{Box 3 Techniques for discovering medication use}

- Normalising non-adherence: 'I often forget to finish a course of antibiotics'

- Testing the patient's knowledge of their medication against the records

- Working to have a trusting and cooperative relationship with the patient, in which honesty feels right

- Requesting considerable detail of routines for taking medicine

- Counting pills and checking against records

- Appealing to the wisdom of knowing true intake if future dosage is to be planned rationally

- Checking general practitioner's records
'I've certainly become more comfortable over the years with coping with the anxiety of my patient saying "No, I'm not going to take my medication doctor because I don't think I need it'.

The extract below illustrates an attempt to promote an honest disclosure of non-adherence by normalising the experience, with the use of brief self-disclosure:

' "OK, so you missed it - well, we all miss things. That's OK - let's see how we can help you stay on it if that's what you want to do" ... you've got to save face'.

Boxes 3 and 4 summarise techniques described by psychiatrists for detecting and managing nonadherence. None of the psychiatrists we interviewed reported using formal compliance therapy (Kemp et al, 1996), nor was this said to be employed by other members of the community mental health team (CMHT). However, methods of interview that corresponded with techniques that might be included in compliance therapy were used:

'Just keep up a low-key discussion of the pros and cons and use motivational interviewing type of thing'.

Some more specific techniques are illustrated below and include exploring the patient's ambivalence to taking medication:

'Patients can feel that their autonomy, their independence as human beings, is being undermined by the use of tablets, which also is quite understandable and is something we should discuss'.

When identifying the drawbacks of medication, this psychiatrist discussed the meaning of taking it and, in particular, the experience of stigma:

'Similarly with drugs, that they don't like being on it, that they're not well motivated to be on it, that they don't like side-effects, that they resent a label of psychiatric illness, that they feel stigmatised, that they want to be "normal"'.

The promotion of self-efficacy through medication is illustrated in the extract below:

'It's important that the patient doesn't blank me out because ... it's just reinforcing the sense of having no control of their lives, which psychosis is already imposing on them ... It's about giving a perspective, a sense of, it's their life, their choice, but they are taking a risk, and do they know what risk they're taking?'.

The metaphor of medication as a coping mechanism is illustrated as follows:

'Very often I say to people, what is their view of the medication? Is it something that is doing something to them or do they see that as something they use to help them, to stay well? So that actually it becomes a coping mechanism, taking the medication is a coping mechanism, rather than a must do or an imperative,

\section{Box 4 Ways of improving adherence}

\section{Prevention}

- Develop a close working relationship, a therapeutic alliance, so that patients will understand why they need medications

- Ask about side-effects regularly

Psychological strategies

- Ask the patient why they are non-adherent and address their reasons

- Normalise the taking of medication

- Encourage a more positive view of medication as a means of coping

- Help patients to perceive a link between not taking medication and becoming unwell

Alternatives to medication strategies

- Explore non-pharmacological treatments

- Agree that the patient has a right not to be medicated

- Accept the fact that the patient does not want medication, and try to maintain contact and communication

- Wait until the patient becomes unwell

Other techniques

- Seek help from other members of the mental healthcare team: pharmacists can give neutral or unbiased information about medication, community psychiatric nurses may act as advocates for patients, to reduce conflict over medication

- Involve relatives in monitoring and encouraging adherence

- Obtain benefits for those who cannot afford their prescriptions 
something you do, or have to do. So you try and build up a sense of mastery, using it as a coping mechanism'.

\section{Conclusions}

Our study identified some common themes. First, that consultant psychiatrists view patient-centred practice as fundamental to encounters with patients involving decisions about the prescription of antipsychotics. They are aware that decisions are made jointly, except in extreme circumstances, and even then they assume sole responsibility for decisions on a temporary basis, being acutely aware of possible damage ensuing to the therapeutic alliance. They are aware of the need to develop the therapeutic alliance and have many differing strategies to enhance it and uncover problems with medication adherence. They are in less agreement about the discussion with patients of their diagnosis and disclosure of side-effects of antipsychotics, and some report using controversial practices such as being mildly untruthful to establish a therapeutic alliance.

The therapeutic alliance is an area in which psychiatrists have developed skills that reflect the particular conditions of the specialty. These specialised skills are passed down to medical students and trainees but are rarely explicitly shared between consultant colleagues, who appear to work in relative isolation (Spurrell, 2000). Further exploration is needed of the sharing of skills among consultants to improve their encounters with patients and of their personal influence on patient outcomes. Consultants should also make use of opportunities to become involved with and learn from other disciplines within the CMHT and to learn from patient feedback via audit or 360-degree appraisal. The next generation of consultant psychiatrists is likely to be at a considerable advantage, with the increasing use of workplace-based assessments in training (Brown \& Doshi, 2006), which is a more valid way of measuring ability to relate to patients. We hope that this account of working practices will assist in promoting the development of therapeutic alliances in psychiatric consultations.

\section{Declaration of interest}

The study from which the examples of interactions are drawn was funded by a grant from Eli-Lilly.

\section{References}

Bhugra, D. \& Holsgrove, G. (2005) Patient-centred psychiatry. Training and assessment: the way forward. Psychiatric Bulletin, 29, 49-52.
Brown, N. \& Doshi, M. (2006) Assessing professional and clinical competence: the way forward. Advances in Psychiatric Treatment, 12, 81-89.

Chaplin, R. \& Kent, A. (1998) Informing patients about tardive dyskinesia. Controlled trial of patient education. British Journal of Psychiatry, 172, 78-81.

Department of Health (2003) Copying Letters to Patients: Good Practice Guidelines. Department of Health.

Frank, A. \& Gunderson, J. (1990) The role of the therapeutic alliance in the treatment of schizophrenia: relationship to course and outcome. Archives of General Psychiatry, 47, 228236.

Gask, L., Dowrick, C., Dixon, C., et al (2004) A pragmatic cluster randomized trial of an educational intervention for GPs in the assessment and management of depression. Psychological Medicine, 34, 63-72.

Kemp, R., Hayward, P., Applewhaite, G., et al (1996) Compliance therapy in psychotic patients: randomised controlled trial. $B M J, 312,345-349$.

Lacro, J. P., Dunn, L. B., Dolder, C. R., et al (2002) Prevalence of and risk factors for medication nonadherence in patients with schizophrenia: a comprehensive review of recent literature. Journal of Clinical Psychiatry, 63, 892-909.

Laugharne, J., Davies, A., Arcelsus, J., et al (2004) Informing patients about tardive dyskinesia: a survey of clinicians' attitudes in three countries. International Journal of Law and Psychiatry, 27, 101-108.

McCabe, R. \& Priebe, S. (2004) The therapeutic relationship in the treatment of severe mental illness: a review of methods and findings. International Journal of Social Psychiatry, 50, 115-128.

McCabe, R., Heath, C., Burns, T., et al (2002) Engagement of patients with psychosis in the consultation: conversation analytic study. BMI, 325, 1148-1151.

Olofinjana, B. \& Taylor, D. (2005) Antipsychotic drugs information and choice: a patient survey. Psychiatric Bulletin, 29, 369-371.

Priebe, S. \& Gruyters, T. (1993) The role of the helping alliance in psychiatric community care: a prospective study. Journal of Nervous and Mental Disease, 181, 552-557.

Priebe, S. \& Gruyters, T. (1999) A pilot trial of treatment changes according to schizophrenic patients' wishes. Journal of Nervous and Mental Disease, 187, 441-443.

Seale, C., Chaplin, R., Lelliott, P., et al (2006) Sharing decisions in consultations involving anti-psychotic medication: a qualitative study of psychiatrists' experiences. Social Science and Medicine, 62, 2861-2873.

Smith, S. \& Henderson, M. (2000) What you don't know won't hurt you. Information given to patients about the side-effects of antipsychotic drugs. Psychiatric Bulletin, 24, 172-174.

Spurrell, M. (2000) Consultant learning groups in psychiatry. Report on a pilot study. Psychiatric Bulletin, 24, 390-392.

Tattan, T. \& Tarrier, N. (2000) The expressed emotion of case managers of the seriously mentally ill: the influence of expressed emotion on clinical outcomes. Psychological Medicine, 30, 195-204.

\section{MCQs}

1 As regards relationships between doctors and patients:

a there is good research showing that training of psychiatrists improves their consultation styles

b research supports the view that patients with psychosis do not want to talk about psychotic symptoms

c a positive therapeutic alliance can improve clinical outcome for a patient with a diagnosis of schizophrenia

$\mathrm{d}$ it is of crucial importance that the doctor and patient are in agreement about the diagnosis

e shared decision-making refers to the sharing of the treatment decision between the psychiatrist and other professional team members. 
2 Techniques that could be effective in developing a therapeutic alliance include:

a adopting a coercive manner

$b$ the use of humour

c using technical language

d excluding disagreement

e showing frequent disapproval of the patient's behaviour.

3 As regards making decisions about the prescription of antipsychotic medication:

a this should only be shared between the psychiatrist and care coordinator

b this should avoid the involvement of carers or other team members

c it might be appropriate to abandon shared decisionmaking when patients lack capacity or are presenting high levels of risk

d shared decision-making is normally abandoned for the duration of treatment under the Mental Health Act

e the abandonment of shared decision-making is usually a long-term treatment decision.

4 Techniques for discovering or managing nonadherence by patients with psychosis living in the community could include:

a attempting to trick a patient into admitting nonadherence

b enquiring in detail about the patient's routine of how they take their medication

c expressing one's irritation to the patient, as it is important to be honest with them

d having a frank disagreement with the patient for therapeutic gain

e covert administration of medication.
5 As regards side-effects:

a patients correctly identify side-effects, making it seldom necessary to enquire about them specifically

$\mathrm{b}$ psychiatrists are in agreement that it is necessary to explain all the potential side-effects before initiating a prescription of antipsychotic medication

c research has shown that patients informed about the risk of tardive dyskinesia are at significantly higher risk of relapse because of non-adherence

d psychiatrists are in agreement about the most appropriate time to disclose to the patient potential side-effects

e under certain circumstances, psychiatrists suggest that obtaining informed consent for an initial prescription may be deferred to a later date.

\section{MCQ answers}

\begin{tabular}{|c|c|c|c|c|}
\hline 1 & 2 & 3 & 4 & 5 \\
\hline a $F$ & a F & a F & a $F$ & a F \\
\hline b F & $\mathrm{b} \mathrm{T}$ & b F & $\mathrm{b} T$ & $b F$ \\
\hline c $\mathrm{T}$ & C F & c $\mathrm{T}$ & c F & c F \\
\hline d F & d F & d F & d F & d F \\
\hline e $F$ & e $F$ & e $F$ & e F & e $\mathrm{T}$ \\
\hline
\end{tabular}

